\title{
Forward-looking perspective on publishing in drug discovery
}

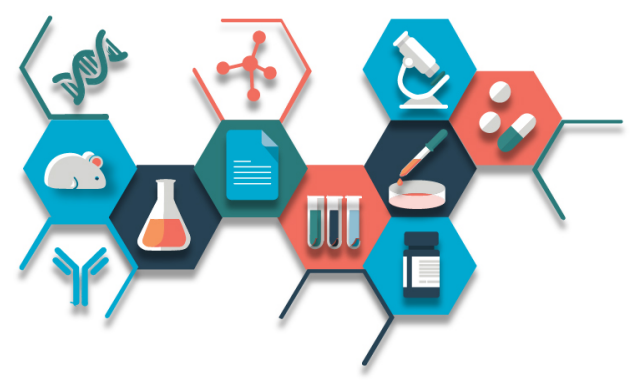

\author{
Jürgen Bajorath ${ }^{*, 1}$ \\ ${ }^{1}$ Department of Life Science Informatics, B-IT, LIMES Program Unit Chemical Biology \& Medicinal Chemistry, Rheinische \\ Friedrich-Wilhelms-Universität, Endenicher Allee 19c, D-53115 Bonn, Germany \\ *Author for correspondence: Tel.: +49 2287369 100; Fax: +49 2287369 101; bajorath@bit.uni-bonn.de
}

\author{
"Hence, there are good reasons for pharmaceutical companies not to abandon publications, \\ despite cultural barriers, and to challenge their scientists to do high-caliber research and expose \\ their work to external review and quality control."
}

First draft submitted: 24 January 2019; Accepted for publication: 25 January 2019; Published online: 20 March 2019

Keywords: academic-industry collaborations $\bullet$ data-driven approaches $\bullet$ data sharing $\bullet$ discovery technologies $\bullet$ drug discovery $\bullet$ intellectual property $\bullet$ interdisciplinary research $\bullet$ open access publishing $\bullet$ restricted open source models

With Future Drug Discovery, a new open access journal enters the field. The new online journal aims to provide a forum for communicating interdisciplinary drug discovery research, from lead generation to the clinic, and for monitoring the discovery field as it further evolves.

No doubt, launching a drug discovery journal is an ambitious endeavor, given the many intellectual property (IP) constraints in pharmaceutical research and its restricted publication culture (see below). There have been a couple of earlier attempts to establish open access publications focused on drug discovery, with very little success. However, the times are changing and so are pharmaceutical companies and the drug discovery field as a whole. Accordingly, the introduction of a new open access journal covering the entire spectrum of drug discovery research and technologies is thought to be rather timely, for reasons further discussed in the following.

\section{Publication culture}

Drug discovery work is notoriously difficult to publish. In the pharmaceutical industry, securing IP positions takes center stage and early disclosure of new chemical entities and discovery research is usually prohibitive. In many instances, drug discovery projects are only reported after they have been discontinued internally. There are various reasons that may lead to discontinuation of discovery projects and failure of drug candidates [1,2]. Termination might be due to lack of efficacy of candidates, toxicity, de-prioritized targets and therapeutic areas, or it might be purely business driven. Regardless, interdisciplinary drug discovery work is a rich and valuable source of knowledge for the field and has generally high priority for publication. Typically, much can be learned from drug discovery case studies. For example, in the Journal of Medicinal Chemistry, project 'stories' from the pharmaceutical industry describing the identification, optimization and preclinical evaluation of candidate compounds are consistently among the most highly accessed publications. Essentially all of these projects have been discontinued or are reported after IP positions have been secured, which typically results in a long delay.

Being an essential part of drug discovery, its IP-driven culture also has downsides. For example, an unnecessary aura of secrecy is frequently generated around any form of discovery research, which hinders scientific communication of results at all levels. Moreover, given current reward and compensation structures in the pharmaceutical industry [3], drug discovery practitioners are preferentially rewarded for successful work in project teams, high throughput and the generation of IP, but not necessarily for publications, depending on the company environment. This likely leads to a lack of entrepreneurial spirits and innovation [3]. In fact, discovery scientists often state that they are simply too busy to write papers, given the many tasks at hand. However, in this context, it is usually not sufficiently considered that publishing under peer review is essentially the only available external control mechanism for scientific standards. Hence, publishing should also be a must for investigators in pharmaceutical research in 
order to position their work in the field, further improve their science and maintain a competitive edge. Hence, there are good reasons for pharmaceutical companies not to abandon publications, despite cultural barriers, and to challenge their scientists to do high-caliber research and expose their work to external review and quality control. High scientific standards and competitiveness are at the heart of innovation and productivity, which is not always appreciated by pharmaceutical companies. In fact, if an aura of self-imposed secrecy in drug discovery settings is taken as an excuse to avoid publication efforts and external scientific discourse, discovery scientists will not further improve their abilities and sooner or later lose their competitive edge. Consequently, things will go into a fundamentally wrong direction, which cannot be in the interest of the pharmaceutical industry.

Hence, from more than one point of view, there is a need for quality journals focusing on drug discovery research and as such Future Drug Discovery is a more than welcome addition to the field.

\section{Journal landscape}

In light of the above, it is not surprising that leading high-impact journals in the area of drug discovery such as Nature Reviews Drug Discovery or Drug Discovery Today are strongly focused on review-type publications. Clearly, reviews are easier to publish in drug discovery than original research. In addition to reviews, medicinal chemistry projects yielding active small molecules or drug candidates are preferentially published in the Journal of Medicinal Chemistry or, occasionally, in Nature or Science journals if the findings have breakthrough character. Then, there are pharmacology publications and reports of clinical studies in various journals. Currently missing is a dedicated forum for communicating early phase interdisciplinary discovery research, also including biologics, and new drug discovery technologies. Conceptually, Future Drug Discovery is well positioned to fill this void.

\section{Recent trends}

A number of recent developments are beginning to change drug discovery and will inevitably lead to more openness and the need to more frequently communicate scientific findings. First, drug discovery is no longer exclusive to the pharmaceutical industry since academic discovery centers and consortia have been founded $[4,5]$. For funding of drug discovery efforts in academia, dissemination of scientific results is essential. Moreover, pharmaceutical companies have started to recognize the value of data sharing and initiating collaborative efforts [6]. Similarly, in recent years, the pharmaceutical industry has increasingly opened up to academia to gain access to basic research, novel targets and new technologies, which has given rise to open innovation strategies and a variety of industryacademic partnerships [6-8]. Despite generally limited drug development capacity in academia, industry-academic collaborations and academic discovery efforts are valuable because they make it possible to target rare or neglected diseases $[9,10]$ that could not be addressed in a profitable way by commercial entities.

In the pharmaceutical industry, these developments gradually foster a more open attitude toward data sharing, integration and communication of scientific results. Currently, we also witness a paradigm shift in pharmaceutical research, with increasing emphasis on unconventional targets such as RNA [11] and biologics as drugs such as recombinant proteins and especially antibodies [12], similar to what was observed during the 1990s. While such developments are cyclical in nature - and expected to change again - they further catalyze interdisciplinary research in drug discovery, trigger a shift from chemistry to biology and lead to a widespread adaptation of new methods and technologies; just to name precise genome editing for target identification and validation as an example [13].

Taken together, these recent trends will likely lead to more open discovery environments and result in more publishable research. Without doubt, communicating new drug discovery technologies and interdisciplinary strategies will be as important as reporting new compounds or biologics. Genetic and sequencing technologies providing the foundation of personalized medicine might serve as a good example [14].

\section{Open source \& open access publishing}

For a rapid and wide dissemination of scientific results, the importance of open source and open access publishing should be strongly emphasized. Together with the rise of online journals, open access publications and open science strategies play an increasingly important role in all areas of science including the life sciences [15]. In addition, open source publications ensure data availability and improved transparency of research activities and thus work against the reproducibility crisis that plagues many scientific fields [16,17]. Especially for data-driven approaches, which rapidly enter the life sciences and drug discovery $[18,19]$, this is of critical relevance.

On the other hand, open source and open access publication models are still not consistently accepted, especially in conservative fields such as pharmaceutical research. While there are strong proponents of open access publishing 
(including the author), others still shy away from associated fees (perhaps forgetting that they or their institutions are required to pay for access to journals). Another problem that works against general acceptance of open access publishing is the flurry of questionable (if not shady) open access journals that have appeared on the scene [20] (and will likely disappear again). Predatory publishing poses a threat to the reputation of open access models and affects serious publishers trying to establish high-quality open access platforms.

For publishing in drug discovery, there is another critically important point to consider, which at least partly conflicts with open source philosophies. Simply put, for drug discovery publications, full data disclosure will not always be possible. Gradual changes toward a more open and publication-friendly attitude in pharmaceutical research do not ensure the ability to fully disclose and deposit all data. This will remain a major issue for internal approval of publications in pharmaceutical companies as pre-patent data disclosure of new therapeutic agents, indications, and associated targets will generally be highly restricted. For discovery methods and protocols, these constraints might often be less severe. Regardless, for publishing in drug discovery, this issue will require consideration. It is advisable to make editorial decisions about the required extent of data disclosure on a case-by-case basis, also taking into account the scientific value and likely impact of a contribution, rather than to implement and enforce hard rules. A certain degree of flexibility concerning data disclosure and deposition requirements will likely be a prerequisite for consistently receiving high-quality submissions from the industry. Otherwise, interesting publications might be prevented, which would be unfortunate for authors, readers and the journal.

While views about the value of peer-reviewed publications still differ in commercial drug discovery settings, offering a variety of paper categories also including case studies, institutional profiles and reviews, as is done by Future Drug Discovery, will help to balance internal restrictions and facilitate discovery publications.

\section{Outlook}

The introduction of Future Drug Discovery is an exciting and important event for the drug discovery community, both in the pharmaceutical industry and academia. The new journal fills a void in drug discovery for open access publication of original research. Moreover, as the pharmaceutical industry is beginning to develop a more open attitude toward research, data sharing, academic collaborations and innovation, open access publications will become increasingly important. There are first encouraging indications. For example, Shire, which is now a part of Takeda, has recently announced the implementation of an open access policy for publication of company-supported research [21]. It is anticipated that such initiatives will have a pilot function.

To make the new journal a success, focusing on interdisciplinary work covering the entire spectrum of earlyphase discovery will be essential, as has been well recognized. Putting emphasis on methodological papers and drug discovery technologies is also highly recommended. Such publications typically spark interests for many investigators in interdisciplinary research settings and the potential impact of these publications is strongly supported by open access models.

Clearly, a new journal offers new opportunities and the author wishes the editor and editorial staff of Future Drug Discovery all the best for the exciting road ahead.

\section{Financial \& competing interests disclosure}

The author has no relevant affiliations or financial involvement with any organization or entity with a financial interest in or financial conflict with the subject matter or materials discussed in the manuscript. This includes employment, consultancies, honoraria, stock ownership or options, expert testimony, grants or patents received or pending, or royalties.

No writing assistance was utilized in the production of this manuscript.

\section{Open access}

This work is licensed under theAttribution-NonCommercial-NoDerivatives 4.0 Unported License. To view a copy of this license, visit http://creativecommons.org/licenses/by-nc-nd/4.0/

\section{References}

Papers of special note have been highlighted as: $\bullet$ of interest; $\bullet \bullet$ of considerable interest

1. Kennedy T. Managing the drug discovery/development interface. Drug Discov. Today 2(10), 436-444 (1997).

2. DiMasi JA. Risks in new drug development: approval success rates for investigational drugs. Clin. Pharmacol. Therap. 69(5), 297-307 (2001). 
3. Douglas FL, Narayanan VK, Mitchell L, Litan RE. The case for entrepreneurship in R\&D in the pharmaceutical industry. Nat. Rev. Drug Discov. 9(9), 683-689 (2010).

- Discussion of reward structures in the pharmaceutical industry and consequences for internal innovation.

4. Frearson J, Wyatt P. Drug discovery in academia: the third way? Expert Opin. Drug Discov. 5(10), 909-919 (2010).

5. Huryn DM. Drug discovery in an academic setting: playing to the strengths. ACS Med. Chem. Lett. 4(3), 313-315 (2013).

6. Khanna I. Drug discovery in pharmaceutical industry: productivity challenges and trends. Drug Discov. Today 17(19-20), 1088-1102 (2012).

7. Bianchi M, Cavaliere A, Chiaroni D, Frattini F, Chiesa V. Organisational modes for open innovation in the bio-pharmaceutical industry: an exploratory analysis. Technovation 31(1), 22-33 (2011).

8. Nilsson N, Felding J. Open innovation platforms to boost pharmaceutical collaborations: evaluating external compounds for desired biological activity. Future Med. Chem. 7(14), 1853-1859 (2015).

9. Coles LD, Cloyd JC. The role of academic institutions in the development of drugs for rare and neglected diseases. Clin. Pharmacol. Therap. 92(2), 193-202 (2012).

10. Ferrins L, Pollastri MP. The importance of collaboration between industry, academics, and nonprofits in tropical disease drug discovery. ACS Infect. Dis. 4(4), 445-448 (2017).

11. Warner KD, Hajdin CE, Weeks KM. Principles for targeting RNA with drug-like small molecules. Nat. Rev. Drug Discov. 17(8), 547-558 (2018).

- Comprehensive perspective on RNA as a drug target.

12. Kinch MS. An overview of FDA-approved biologics medicines. Drug Discov. Today 20(4), 393-398 (2015).

13. Moore JD. The impact of CRISPR-Cas9 on target identification and validation. Drug Discov. Today 20(4), 450-457 (2015).

14. Goodwin S, McPherson JD, McCombie WR. Coming of age: ten years of next-generation sequencing technologies. Nat. Rev. Genet. 17(6), 333-351 (2016).

15. McKiernan EC, Bourne PE, Brown CT, et al. Point of view: how open access helps researchers succeed. Elife 5, e16800 (2016).

16. Baker M. Is there a reproducibility crisis? Nature 533(7604), 452-454 (2016).

-. Extensive survey of current (lack of) reproducibility in science.

17. Hossenfelder S. Science needs reason to be trusted. Nat. Phys. 13(4), 316-317 (2017).

18. Lusher SJ, Ritschel T. Finding the right approach to big data-driven medicinal chemistry. Future Med. Chem. 7(10), 1213-1216 (2015).

- Discussion of opportunities for data-driven medicinal chemistry in drug discovery.

19. Bajorath J, Jenkins J, Overington J, Walters WP. Drug discovery and development in the era of big data. Future Med. Chem. 8(15), 1807-1813 (2016).

-. Different views of data challenges and opportunities in drug discovery.

20. Butler D. The dark side of publishing. Nature 495(7442), 433-435 (2013).

-. Instructive account of predatory publishing.

21. Shire. (2019) www.shire.com/en/newsroom/2018/january/xajhds 